\title{
La percepción de la conducta agresiva: Un estudio taxonómico de las diferencias inter e intraculturales
}

\author{
Jesús Varela Mallou, \\ José Manuel Sabucedo Cameselle, \\ Constantino Arce Fernández \\ Universidad de Santiago de Compostela

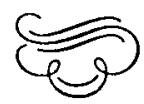 \\ Resumen
}

El presente artículo tiene como principal objetivo ser una alternativa a las tradicionales clasificaciones teóricas de la agresión e, incluso, a los estudios taxonómicos empiricos más recientes. Usar estímulos no representativos, ofrecer indicios sobre las dimensiones de juicios preseleccionados, etc. son algunos de los inconvenientes que no permiten considerar a estas dimensiones perceptuales de la agresión como fiables. En este trabajo se pretenden superar estas y otras criticas con el fin de proporcionar las verdaderas dimensiones que subyacen a la percepción social de los episodios agresivos cotidianos. El Escalamiento Multidimensional (Modelo INDSCAL) fue seleccionado como el análisis más apropiado para proponer una taxonomía empírica adecuada y comprobar hasta qué punto una misma taxonomía es compartida por diferentes subculturas (Universitaria y Reclusa). El análisis de los datos nos permitió concluir acerca de la existencia de un dominio psicológico en 4 dimensiones para percibir un conjunto de estímulos agresivos, de la existencia de ciertas diferencias entre ambas submuestras y, por último, de la bomogeneidad existente dentro de una misma muestra, independientemente de algunas variables de personalidad previamente seleccionadas.

Palabras clave: Escalamiento multidimensional, taxonomía empírica, episodio agresivo, dimensiones y subcultura.

\section{Abstract}

This study empirically attempts to offer contributions for the classification of aggressive episodes. The proximity data approach is followed. Under this perspective, aggressive episodes are mapped as points in a n-dimensional space. Data were collected for two different spanish subcultures: undergraduates and prisoners. An individual differences multidimensional escaling model and method (Carroll and Chang, 1970) was used to analyze the data. The four-dimensional solution was retained. Differences between subcultures are found.

Key words: Multidimensional scaling, taxonomie, aggressive episode, dimension and subculture.

Dirección de los autores: Departamento de Metodología. Facultad de Psicología. Universidad de Santiago de Compostela. La Coruña. España. 


\section{INTRODUCCION}

Una aproximación, aunque sea superficial, al tema de la agresión permite comprobar a cualquier lector interesado en estas cuestiones, el enorme auge de los trabajos realizados en este área (Bandura, 1973; Berkowitz, 1970; Birhbacher, 1984; Buss, 1961; Felson, 1982; Lorenz, 1966; Tedeschi, 1983, etc.). Sin embargo, la proliferación de teorías y modelos no se ha traducido en una mayor clarificación del concepto a estudiar. Tal vez, esta falta de acuerdo sea la que llevó a muchos autores a aceptar que la agresión no tiene por qué ser un concepto «unitario» pudiendo, por lo tanto, existir distintos tipos o clases de agresión. Fue por ello que, desde hace unos años, los psicólogos se preocuparon por identificar las distintas modalidades existentes de la conducta agresiva convencidos que, de este modo, aumentarían la homogeneidad entre los distintos tipos de agresión y se podría consensuar una tipología de la misma.

Si bien este tipo de investigaciones taxonómicas fueron muy prolíficas, ello no fue suficiente para que la ciencia psicológica disponga, hoy en día, de una adecuada y única taxonomía que ponga un poco de orden y permita clasificar, de forma más homogénea, los distintos actos agresivos. La dificultad de encontrar una taxonomía compartida quizás se daba a que, todas ellas, están sesgadas por las predilecciones de la posición teórica en que se sitúa cada autor, en argumentos especulativos o en extrapolaciones desde las teorías existentes.

Al respecto, Berkowitz (1962, 1969), Toch (1969), Gunn (1973), entre otros, señalaron que, a pesar de la voluminosa literatura sobre agresión, se conoce poco acerca de las distinciones que hacen las personas entre actos agresivos cotidianos. Forgas, Brown y Menyhart (1980) y Campbell, Muncer y Bibel (1985) contribuyeron a estas reflexiones estudiando las percepciones legas de los episodios agresivos para, de este modo, construir lo que denominamos taxonomías empíricas (en contraposición a las anteriores denominadas teóricas).

Más concretamente, Campbell et al. (1985) después de una extensa revisión teórica, propusieron una taxonomía ecléctica basada en los Motivos (instrumental, hostil, normativo y elevador de estatus) y la Forma (directa, indirecta, física y verbal) y la ratificaron empíricamente. Aunque ellos mismos advertían del carácter preliminar del estudio, todo parecía indicar que sería una clasificación relevante en cuanto recogía los distintos marcos teóricos y, además, había sido comprobada empíricamente.

No obstante, en un estudio posterior, Varela, Arce y Sabucedo (1989) pusieron de manifiesto la falta de acuerdo entre esta taxonomía y las distinciones realizadas por las muestras de sujetos legos. Dichos resultados señalaron la necesidad de continuar investigando sobre las verdaderas dimensiones que subyacen a la percepción de los episodios agresivos. Será, por tanto, el que aquí se realice, un estudio de tipo exploratorio.

Antes de introducirnos en la presente investigación, se consideró conveniente exponer alguno de los antecedentes más relevantes. Así, mientras que Attilli y Hinde (1986) proponen una clasificación a partir de las elaboradas por otros autores (reconociendo cuatro categorías generales: instrumental, hostil, injurias e insultos y hostilidad defensiva), Henderson (1986) realiza su clasificación atendiendo a la situación donde sucede la agresión, por lo que 
distingue entre agresión familiar o doméstica y si se lleva a cabo en lugares públicos o privados.

- Por su parte, Sherman y Dowdle (1974) analizan las percepciones de los delitos y castigos en sujetos universitarios y, utilizando la técnica de Escalamiento Multidimensional (MDS), encontraron que se requiere un espacio en cuatro dimensiones. Toch (1969) habla de agresión provocada y no provocada, Schott (1971) distingue también dos tipos: planeada y no planeada, Buss (1961): agresión verbal, física y directa; etc.

Desde una perspectiva empírica, Forgas et al. (1980) usaron el Escalamiento Multidimensional en la investigación de la percepción de los episodios agresivos. Estos autores requerían a los sujetos que calificaran los estímulos agresivos en 12 escalas bipolares, las cuales fueron construidas para incorporar las dimensiones comúnmente defendidas por los teóricos como importantes. $\mathrm{Al}$ proveer tales escalas, los investigadores están ofreciendo pistas de las dimensiones importantes $y$, la solución dimensional resultante, revela muy poco acerca de las verdaderas dimensiones cognitivas implícitas de los sujetos.

Con el fin de superar la crítica realizada al trabajo de Forgas et al. (1980), Campbell et al. (1985) emplearon tareas de evaluación directa de la disimilaridad entre pares de estímulos. Sin embargo, a nuestro entender, cometen un error similar al no utilizar situaciones agresivas representativas (ya que los estímulos agresivos fueron generados por los propios investigadores).

En general, se puede decir que estos estudios previos, poseen algunos inconvenientes de procedimiento: usan como estímulos un subgrupo arbitrario y pequeño del posible rango de episodios agresivos, utilizan episodios agresivos que describen situaciones no experienciadas personalmente por la mayoría de los sujetos, utilizan características y dimensiones de juicios preseleccionados, etc. Es por ello que, las diferencias encontradas entre estímulos, no proporcionan ninguna información fiable sobre las dimensiones perceptuales que subyacen a la representación cognitiva de tales estudios agresivos.

El presente estudio fue diseñado para superar algunos de estos problemas. En vez de imponer las escalas unidimensionales y los estímulos agresivos, se llevó a cabo un estudio piloto normativo con el fin de obtener un grupo de episodios agresivos representativos del medio cotidiano.

Además, se utilizaron dos muestras subculturalmente diferentes (universitarios y reclusos) porque se pretende profundizar en las posibles fuentes de variación de las taxonomías. En concreto, se desea dar respuesta a por qué en un estudio previo (Varela et al. 1989), no se corroboró la taxonomía de Campbell et al. (1985). Para ello, se entendió a la agresión como una secuencia de interacción social que denominamos episodio agresivo y que, por suceder en un determinado entorno cultural no puede estar exento de unos componentes afectivos, emocionales y normativos. Desde este punto de vista, en el que la información proveniente de los episodios agresivos incluye sucesos altamente significativos para los sujetos, se admite que los sucesos tienden a covariar manteniendo relaciones de contingencia entre ellos. La percepción de esas contingencias dan lugar a aprendizajes que constituyen la cultura o socialización del sujeto.

Todo ello llevó a plantear que los actos agresivos son percibidos y entendidos en función de los discursos sociales que se han elaborado sobre los mismos dentro de una comunidad determinada. Esto implica que los sujetos, con unos antecedentes dados, pueden compartir una percepción común de los 
episodios agresivos que suceden en su medio cotidiano. Para confirmar esto se controló, en primer lugar, el grupo de referencia para ver si se ajusta a una pauta intercultural (pudiendo explicar así los resultados encontrados en Varela et al., 1989), o bien, si está mediatizada por las diversas variables actitudinales y de personalidad.

El Escalamiento Multidimensional (MDS), fue seleccionado como el análisis más apropiado para esta tarea dada la variedad de recolección de datos que ofrece (Arce, 1989), y porque hace significativos los juicios de comparación de los sujetos sin la intervención de constructos externos por parte del experimentador. En concreto, se utilizó el modelo de las diferencias individuales (INDSCAL) de Carroll y Chang (1970). Este modelo asume que una muestra de individuos usan las mismas dimensiones para juzgar un grupo de estímulos, pero esas dimensiones son diferencialmente relevantes para ellos, incluyendo la posibilidad de relevancia cero. Una ventaja más del INDSCAL es que las dimensiones estimulares no necesitan ser rotadas de nuevo $\mathrm{y}$, normalmente, se «corresponden a dimensiones psicológicas significtivas en un sentido muy estricto» (Carroll y Chang, 1970, 265). En Arce, Seoane y Varela (1989) se analiza el procedimiento matemático y otros aspectos de interés aplicado al modelo INDSCAL y a otros tres modelos que, en opinión de los autores, constituyen la esencia de la teoría del escalamiento multidimensional.

Otra novedad de la presente investigación, con respecto al estudio de Campbell et al. (1985), es que la interpretación de los resultados se llevó a cabo de forma empírica a través de los pesos de las escalas bipolares en el espacio multidimensional seleccionado:

En suma, con el presente estudio se plantearon los siguientes objetivos: (a) encontrar una taxonomía empírica adecuada a través de las características o atributos utilizados por los sujetos para percibir los estímulos agresivos; para ello se utilizó la técnica del Escalamiento Multidimensional; (b) que la taxonomía resultante refleje un grado de acuerdo consensuado en la subcultura previamente definida, y (c) evaluar si las diferentes características actitudinales $y$ de personalidad intervienen en la percepción de los diferentes tipos de episodiso agresivos.

Con todo ello, se espera que los resultados obtenidos permitan dar respuesta a cuestiones como las siguientes: las representaciones de episodios agresivos ¿son estructuralmente diferentes en distintas subculturas? Dado un espacio multidimensional, los mismos episodios agresivos ¿ocupan posiciones similares o diferentes para distintos grupos de sujetos?; si existen diferencias, ¿éstas pueden estar relacionadas con las circunstancias normativas de diferentes grupos o, por el contrario, se deben a diferencias individuales?.

\section{METODO}

\section{Sujetos}

Una vez seleccionados los episodios agresivos y las escalas bipolares, se obtuvieron los juicios de 131 sujetos que decidieron participar voluntariamente en el conjunto de las pruebas realizadas. De estos sujetos, 56 eran estudiantes de la Universidad de Santiago y 75 eran delincuentes encarcelados pertenecientes a las prisiones gallegas de Monterroxo, Bonxe y Provincial de La Coruña. 
Debido a los numerosos días de participación que demandábamos de los sujetos, éstos quedaron reducidos a 43 universitarios y 43 reclusos.

\section{Episodios estimulares y escalas bipolares}

De acuerdo con la orientación conceptual del estudio, se pretendió que los estímulos a escalar fuesen un buen reflejo de todos los posibles episodios agresivos comúnmente experienciados por la muestra objeto de estudio. Por ello, se realizó un estudio normativo en el que participaron 90 estudiantes universitarios y 82 reclusos preventivos y de cumplimiento.

A estos sujetos se les solicitó que describieran episodios agresivos tan personalmente experienciados como pudieran recordar, independientemente de cuándo, dónde y a quién le hubiera sucedido. Naturalmente, no se les proporcionó ninguna definición de agresión ya que el propósito del estudio era examinar la estructura del acto agresivo tal y como lo entienden los sujetos.

De los 430 episodios agresivos recogidos, después de realizar un análisis de contenido, fueron seleccionados los 28 episodios más frecuentemente mencionados por las dos muestras.

En cuanto a las escalas bipolares, éstas fueron construidas a partir del análisis de las respuestas libres de los adjetivos para cada uno de los episodios agresivos descritos anteriormente. De un total de 906 adjetivos, fueron seleccionados 16 en función de los tres criterios que exponemos a continuación: saliencia o frecuencia absoluta de uso a través de los episodios, productividad o diversidad de uso a través de los episodios e independencia o falta de concurrencia entre episodios.

El último paso consistió en buscar un antónimo para cada uno de los 16 adjetivos seleccionados. De este modo, se construyó la lista de adjetivos bipolares siguiendo el método del diferencial semántico de Osgood (1964) (véase primera columna de la Tabla I).

\section{Procedimiento}

Dado que del estudio normativo resultaron seleccionados 28 episodios agresivos, es desaconsejable el procedimiento de evaluación directa de la (di)similaridad (empleado por Campbell et al., 1985 y Varela et al., 1989), ya que supondría un total de 378 pares de combinaciones estimulares. Sin duda, supondría una excesiva demanda de esfuerzo y tiempo para los sujetos. Esta fue una de las razones de por qué, en los anteriores estudios, se utilizó una muestra de estímulos relativamente pequeña, a pesar de la consiguiente pérdida de representatividad.

Con la intención de mantener la representatividad se utilizó una tarea distinta, los datos de perfil. Según este procedimiento, cada uno de los 28 estímulos fueron evaluados en cada una de las 16 escalas bipolares formadas por la lista de adjetivos que suministraron los propios sujetos. La presentación de las escalas bipolares fue realizada según el procedimiento recogido por Davison (1983, 45-47) y, antes de comenzar la tarea, se pidió a los sujetos que leyeran todos los estímulos con el propósito de igualar sus expectativas. Estos 
datos fueron ordenados en una matriz de tres vías de la forma: episodios agresivos $X$ escalas bipolares $X$ sujetos, donde cada fila representa el perfil de un episodio agresivo para un sujeto cualquiera i. Este procedimiento fue originalmente ideado por Wish, Deutsch y Kasplan (1976), tratado en Arce (1989) y utilizado, más recientemente, por Forgas et al. (1980), Arce y Real (1989) y Varela (1989).

Posteriormente, los datos de perfil se transformaron en $\mathbf{i}$ matrices de (di)similaridad simétrica $(\Delta)$ empleando la fórmula:

$$
\delta_{j k(i)}=\left[1 / T \sum_{S=1}^{T}\left(x_{j s(i)}-x_{k s(i)}\right)^{2}\right]^{1 / 2}
$$

donde un elemento cualquiera $\delta_{\text {jk(i) }}$ representa la disimilaridad entre el episodio j y el episodio $\mathrm{k}$ para el sujeto $\mathrm{i}, \mathrm{T}$ es el número total de escalas y $\mathbf{s}$ un escala.

Este procedimiento es de menos demanda y consumo de tiempo para los sujetos; además, dado que es un número razonable de escalas, es improbable que los juicios de los sujetos se vean afectados por las escalas empleadas, ya que las matrices de disimilaridad, usadas como entrada de datos para el INDSCAL, se basan en los juicios de muchos sujetos en 12 escalas. Por otro lado, esto se ve reforzado por el hecho de que las escalas usadas son relevantes para los sujetos.

\section{Medidas de actitudes y personalidad}

Se evaluaron los siguientes rasgos de personalidad: Deseabilidad Social (Crowne y Marlow, 1960), Self-Monitoring (Snyder, 1974), Inventario S-R de Hostilidad (Endler y Hunt, 1968), Psicoticismo, Neuroticismo y Extraversión (Eysenck y Eysenck, 1964), Irritabilidad y Sadismo de Jackson y Messic (1962) y Maquiavelismo de Christie y Geis (1970). Estos cuestionarios fueron aplicados en grupos de 10 sujetos en la muestra universitaria y de 3 a 5 en la de reclusos.

Se utilizaron las instrucciones particulares de cada cuestionario y los resultados se analizaron siguiendo los procedimientos estándar de puntuación, en función de los cuales, se identificó al $25 \%$ de los sujetos con puntuaciones más bajas y al $25 \%$ con puntuaciones más altas para cada medida. Posteriormente, se evaluaron las diferencias en la percepción de episodios agresivos en estos grupos mediante una serie de nueve análisis discriminatorios.

\section{RESULTADOS}

\section{Muestra universitaria}

Con el fin de descubrir la estructura subyacente a los datos, se analizaron las 43 matrices de disimilaridad (una matriz por sujeto universitario) obtenidas por la Fórmula 1, mediante el uso de la versión no-métrica del modelo INDSCAL implementada en el programa ALSCAL de Takane, Young y DeLeeuw (1977).

Se obtuvieron representaciones del espacio perceptual para los episodios agresivos en 6, 5, 4, 3 y 2 dimensiones. La primera tarea, fue decidir el número 


\section{3}

óptimo de dimensiones. Shepard (1972) mantiene que esta decisión «ha de constituir un punto de equilibrio entre la bondad de ajuste, la interpretación y la parsimonia obtenida con la solución». En el presente caso parecía óptima la solución en 4 dimensiones.

En las Figuras I y II se ofrece la configuración de estímulos en 4 dimensiones (presentadas de 2 en 2) con un $\mathrm{STRESS}=.223$ y RSQ $=.83$.

Figura 1

Configuración de estímulos para las Dimensiones I y II. Universitarios.

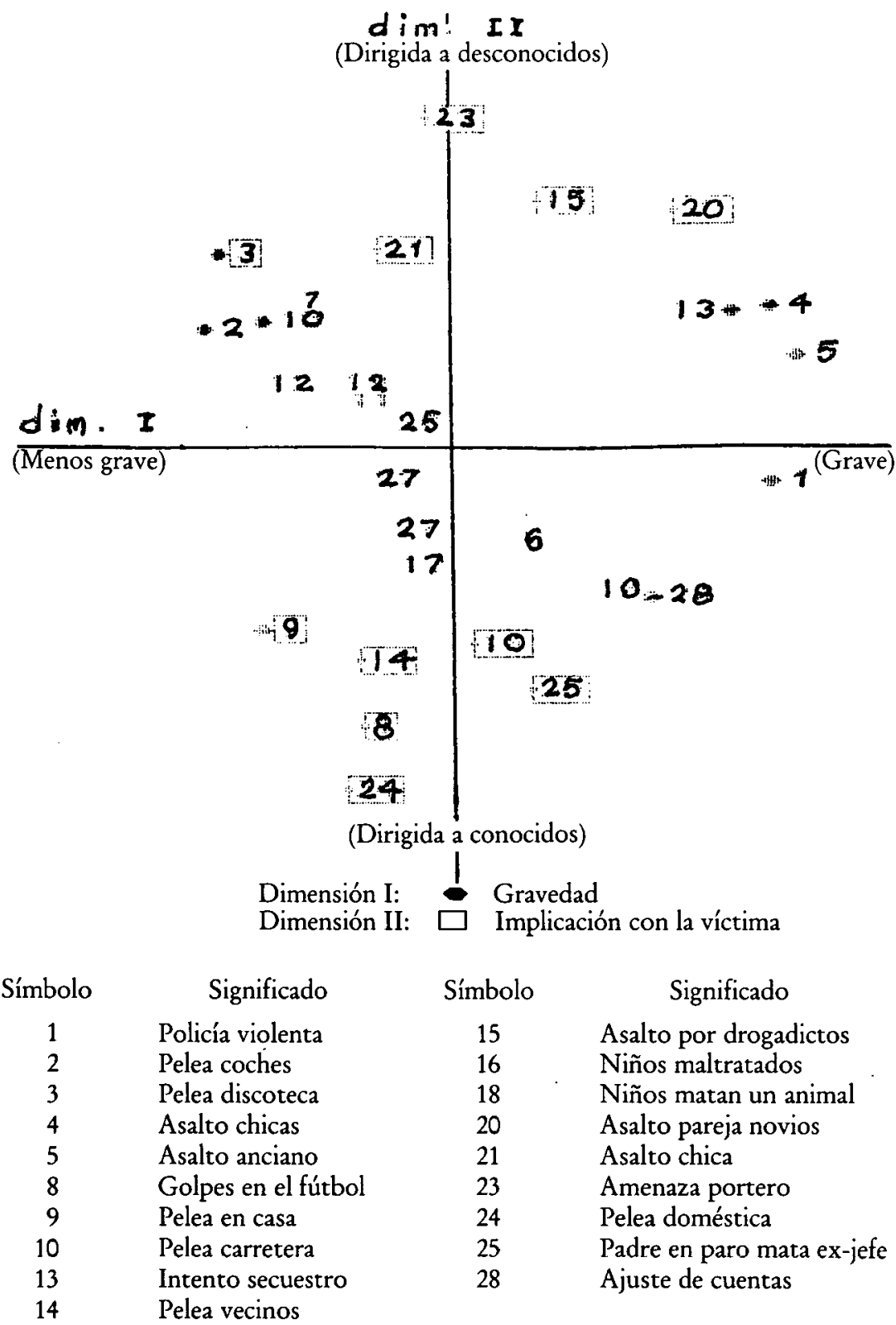


Figura 2

Configuración de estímulos para las Dimensiones III y IV. Universitarios.

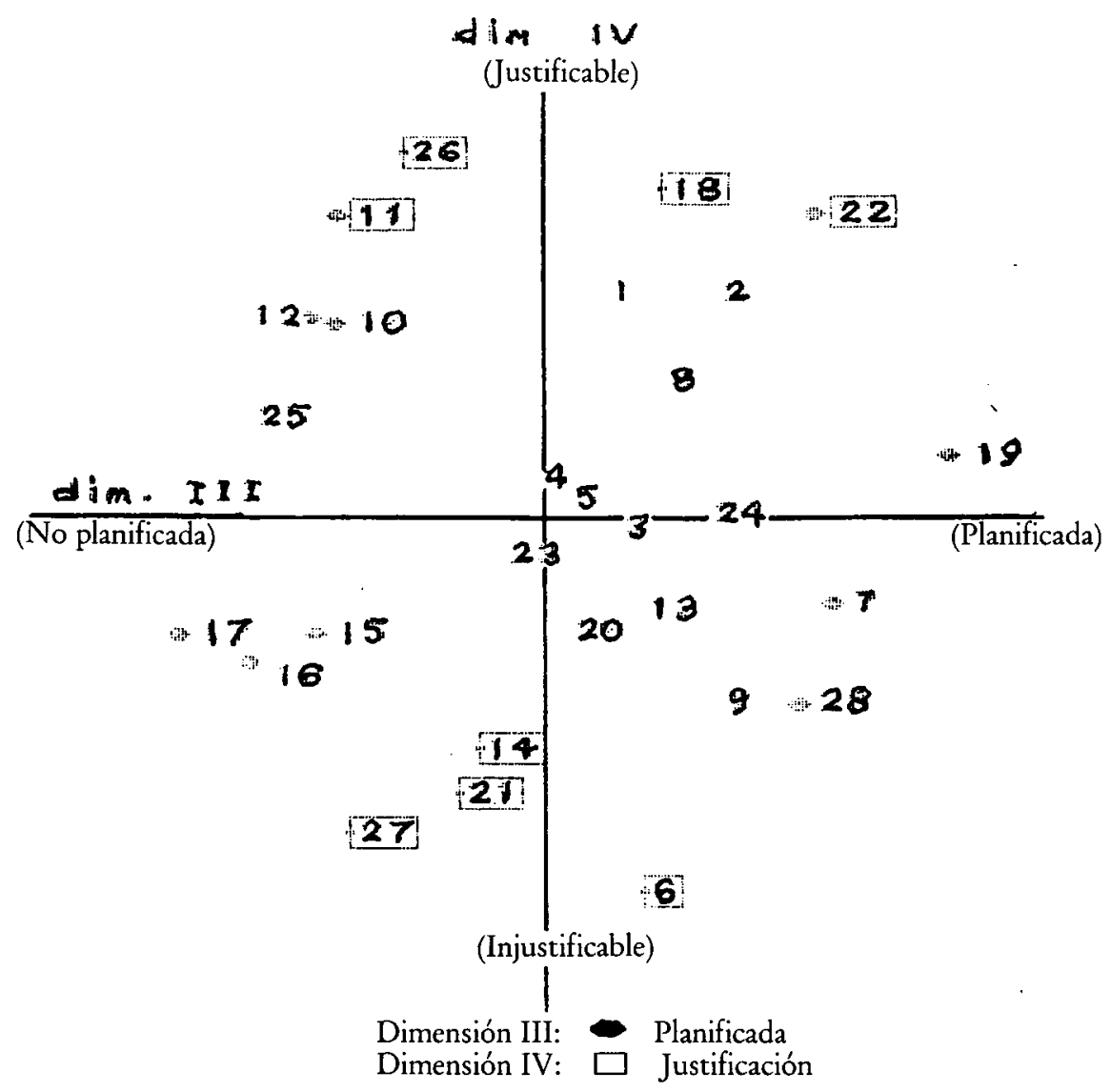

\begin{tabular}{clcl} 
Símbolo & \multicolumn{1}{c}{ Significado } & Símbolo & \multicolumn{1}{c}{ Significado } \\
6 & Violación novio & 17 & Alcohólico maltrata a su mujer \\
7 & Insultos bar & 18 & Niños matan un animal \\
10 & Pelea carretera & 19 & Discusión en el cine \\
11 & Borracho causa pelea & 21 & Asalto chica \\
12 & Pelea bar & 22 & Discusión en el autobús \\
14 & Pelea vecinos & 26 & Pelea en el campo fútbol \\
15 & Asalto drogadictos & 27 & Apuñalar a un compañero \\
16 & Niños maltratados & 28 & Ajuste de cuentas
\end{tabular}

Para facilitar la interpretación de las dimensiones, los datos de perfil anteriores, se transformaron mediante la Fórmula 2, ideada por Wish y Kaplan (1977):

$$
\delta_{\mathrm{jk}(\mathrm{s})}=\left[1 / \mathrm{N} \sum_{\mathrm{i}=1}^{\mathrm{N}}\left(\mathrm{x}_{\mathrm{ij}(\mathrm{s})}-\mathrm{x}_{\mathrm{ik}(\mathrm{s})}\right)^{2}\right]^{1 / 2}
$$


donde $\mathbf{j}$ y $\mathbf{k}$ son dos episodios agresivos, $\mathbf{i}$ es un sujeto, $s$ una escala y $\mathbf{N}$ el número total de sujetos.

Los datos obtenidos fueron 16 matrices de disimilaridad simétricas (una matriz por escala bipolar). Por tanto, la matriz tri-dimensional de datos tendrá la ordenación: episodios agresivos $X$ episodios agresivos $X$ escalas bipolares. La aplicación de las Fórmulas 1 y 2 fue realizada con la ayuda de un programa BASIC construido para tal fin.

Una vez ejecutado un nuevo INDSCAL con las 16 matrices de orden $28 \times 28$, y dado que cada matriz aquí es una escala bipolar, en lugar de un sujeto, la relación entre esas escalas y las dimensiones ofrecidas por el INDSCAL es una buena ayuda para la interpretación de la estructura seleccionada en 4 dimensiones.

A continuación, en la Tabla I, presentamos los pesos de las escalas en cada una de las dimensiones con el fin de conocer cuáles son las que dan sentido a las mismas. Estos pesos se interpretan como coeficientes de correlación parcial entre una escala bipolar conocida y una dimensión estimular del análisis INDSCAL (Arce y Gärling, 1989).

TABLA I

Pesos de las escalas en cada una de las 4 dimensiones.

\begin{tabular}{llcccc}
\hline & & & \multicolumn{3}{c}{ DIMENSIONES } \\
& & I & II & III & IV \\
\hline grave & no grave & $.776+$ & .2001 & .0661 & .0728 \\
justificable & injustificable & .2543 & .1996 & .2264 & $.4204+$ \\
violento & no violento & .2706 & .1 .684 & .1643 & .1568 \\
planeada & no planeada & .2023 & .1046 & $.6287+$ & .0677 \\
justo & injusto & .3169 & .1923 & .2112 & .5412 \\
bebida causante & no causante & .1424 & .1304 & .1578 & .0906 \\
emocional & no emocional & .2113 & .1928 & .3091 & .1219 \\
controlado & incontrolado & .1276 & .2019 & $.5813+$ & .1506 \\
potencia & impotencia & .2930 & .2072 & .2470 & .1756 \\
deseable & indeseable & .2282 & .2029 & .2076 & .2444 \\
tolerable & intolerable & .0767 & .2112 & .2229 & $.5777+$ \\
seguridad & inseguridad & $.5912+$ & .2013 & .1030 & .1809 \\
obtener beneficios & no obtener beneficios & .1082 & .1088 & .1235 & .0765 \\
sociedad responsable & individuo responsable & .0971 & .0989 & .1114 & .0642 \\
dirigida conocidos & no dirigida conocidos & .0483 & $.9485+$ & .0387 & .0809 \\
humillación & no humillación & .2455 & .1872 & .2043 & .1968 \\
\hline
\end{tabular}

Tal y como se observa en la Tabla I, los pesos más altos en la dimensión I, corresponden a las escalas Grave-No grave (.777) y Seguridad-Inseguridad (.591). Estos resultados, junto con el espacio de estímulos (Figura 1), permitieron interpretar esta dimensión como "gravedad de la situación desde el punto de vista de la inseguridad ciudadana». Si se inspecciona visualmente el eje de abcisas de la Figura 1, se observa cómo aquellas formas de agresión que describen asaltos a las personas se sitúan a la derecha (estímulos rodeados con un círculo bajo los símbolos 5, 4, 1, 13, 27). Mientras que, aquellas situaciones menos graves, en cuanto surgen a partir de una interacción social cotidiana, se sitúan a la izquierda (estímulos 2, 3, 10, 9). 
La dimensión II se caracteriza exclusivamente por la escala Dirigida a conocidos-No dirigida a conocidos (.948). Esta dimensión fue interpretada como "grado de implicación con la víctima». En la Figura 1 (eje de ordenadas) se fortalece esta interpretación en cuanto a los estímulos $23,15,20,21$ y 3 se sitúan en la parte superior del eje (todos tuvieron lugar entre personas desconocidas). Por el contrario, los que ocurrieron entre personas conocidas e incluso entre familiares (estímulos 24, 8, 25, 14, 16, 9) se distribuyen en la parte inferior del eje.

En cuanto a la dimensión III (eje de abcisas en la Figura 2) los pesos más altos se corresponden con las escalas Planeada-No planeada (.628) y ControladoIncontrolado (.581). Esta dimensión fue interpretada como "Instrumental versus Hostil» porque, como se observa en la Figura 2, los estímulos con cierta intencionalidad, cometidos para obtener beneficios $(19,7,22,28)$ se sitúan a la derecha y, por el contrario, aquellos otros que pudieron surgir impulsivamente, sin planificación ni control, en donde la conducta agresiva pudiera estar motivada por los resultados injuriosos de la acción, se situaron en la parte izquierda de la dimensión (los estímulos 17, 6, 15, 12, 10, 8).

Por último, en la dimensión IV, se obtuvieron unos pesos elevados en las escalas Tolerable-Intolerable (.577) y Justo-Injusto (.541). Esta dimensión fue interpretada como «justificabilidad en el sentido de tolerabilidad». En la Figura 2, se observa que los estímulos que se sitúan en la parte inferior del eje de ordenadas son calificados de poco justificables y repudiables representando situaciones de violación (estímulo 14), peleas entre vecinos (21), ajuste de cuentas (27), violación por parte del novio (6). Mientras que en la parte superior se agrupan estímulos que pueden ser evaluados como esperados, o incluso como adecuados, si se tiene en cuenta cómo se produjeron. Por ejemplo, una pelea causada por un borracho (26), discusión con el conductor ante el retraso del autobús (M), golpes en el campo de fútbol (22), etc.

De este modo, los dos análisis INDSCAL (sujetos y escalas), han permitido identificar las 4 dimensiones utilizadas por la muestra de sujetos universitarios, a la hora de percibir distintos episodios agresivos (gravedad, implicación con la víctima, instrumentalidad y justificabilidad).

\section{Muestras de reclusos}

Paralelamente al análisis multidimensional realizado con la muestra de universitarios, se llevaron a cabo procedimentos y análisis similares para la muestra derecursos (estudio normativo, obtención de datos de perfil, transformación de éstos en puntuaciones de disimilaridad para sujetos y escalas, análisis INDSCAL e interpretación de las dimensiones).

La selección de la dimensionalidad mínima que se derivó para la muestra de reclusos se llevó a cabo teniendo en cuenta los valores del Stress y RSQ, éstos se analizaron mediante un criterio similar al test de Cattell o «Scree Test» en el análisis factorial. En concreto, fue seleccionada, la solución en tres dimensiones con un Stress asociado de .241 y un RSQ de .79.

Además, esta dimensionalidad resultó útil por ser interpretable empíricamente. Esto es, los pesos de las escalas bipolares en las 3 dimensiones aconsejaron interpretarlas como justificabilidad, instrumental y grado de implicación con la víctima, respectivamente. Dado que en la muestra de 
universitarios habían sido seleccionadas 4 dimensiones, también se requirió al programa la solución en 4 dimensiones y se comprobó la escasa interpretabilidad de la 4. ${ }^{2}$ dimensión; donde la escala bipolar con más peso, en esa dimensión, es de 0.27 .

Sin embargo, dadas las características del modelo INDSCAL ( 3 vías, ponderaciones de los sujetos y espacio común), no es adecuado establecer comparaciones entre dos análisis INDSCAL diferentes para dos muestras independientes. Unicamente se puede inferir que los sujetos universitarios utilizan un espacio en 4 dimensiones para percibir y clasificar los episodios agresivos, mientras que los sujetos encarcelados lo hacen desde un espacio tridimensional.

Dado que la diferencia, entre ambas submuestras, viene dada por el uso o no de la dimensión de "gravedad de la situación», se pensó que es conveniente comprobar en qué medida los sujetos encarcelados conceden más o menos importancia a esta dimensión.

Con el objeto de utilizar un mismo «espacio de sujetos» en primer lugar, se redujeron las 86 matrices de disimilaridad (43 de universitarios y 43 de reclusos) a 2 matrices de disimilaridad promedio (una por grupo cultural). Estas 2 matrices sirvieron de entrada de datos para el programa ALSCAL, del cual se obtuvo una solución en 4 dimensiones (STRESS $=.130, \mathrm{RSQ}=.850$ ). Tras comprobar que los pesos más altos en la dimensión I se correspondían con las escalas bipolares grave-no grave (.665), seguridad-inseguridad (.517), ésta se interpretó como «gravedad de la situación».

A continuación, si se observa el «espacio de sujetos total» en la Figura 3, donde el número 1 representa al promedio de los sujetos universitarios y el número 2 a los reclusos, se concluye (en función de la longitud y dirección de los vectores) que la dimensión de "gravedad de la situación" no posee una especial saliencia para el conjunto de los presos, a la hora de distinguir entre un conjunto de episodios agresivos que suceden naturalmente.

Figura 3

Pesos de los sujetos en la Dimensión I de «Gravedad de la situación»

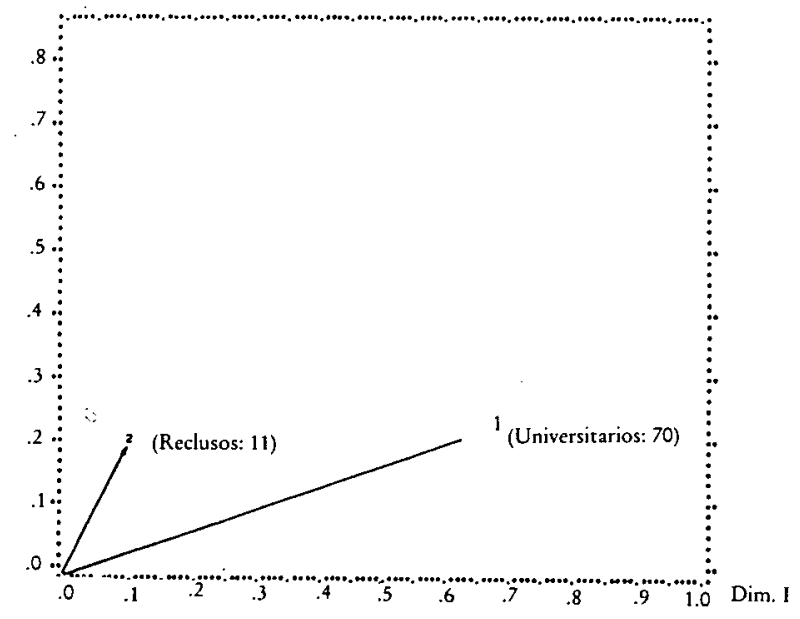


A la luz de los espacios tanto de estímulos como de sujetos, cabría destacar dos aspectos de interés. En primer lugar, que el número de dimensiones utilizadas guarda relación con los antecedentes normativos y subculturales de los sujetos. En segundo lugar, que la variabilidad entre ambas muestras no viene dada tanto por el concepto general de las dimensiones (ya que comparten 3 , y la $4 .^{a}$ parece ligada a un problema de inseguridad ciudadana que los reclusos no padecen), sino por la situación tan distinta que ocupan los episodios agresivos en sus correspondientes "espacios estimulares» para ambas muestras (compárese las Figuras 1 y 2 vs. Figuras 4 y 5 ).

\section{Figura 4}

Configuración de estimulos para las Dimensiones I y II. Reclusos.

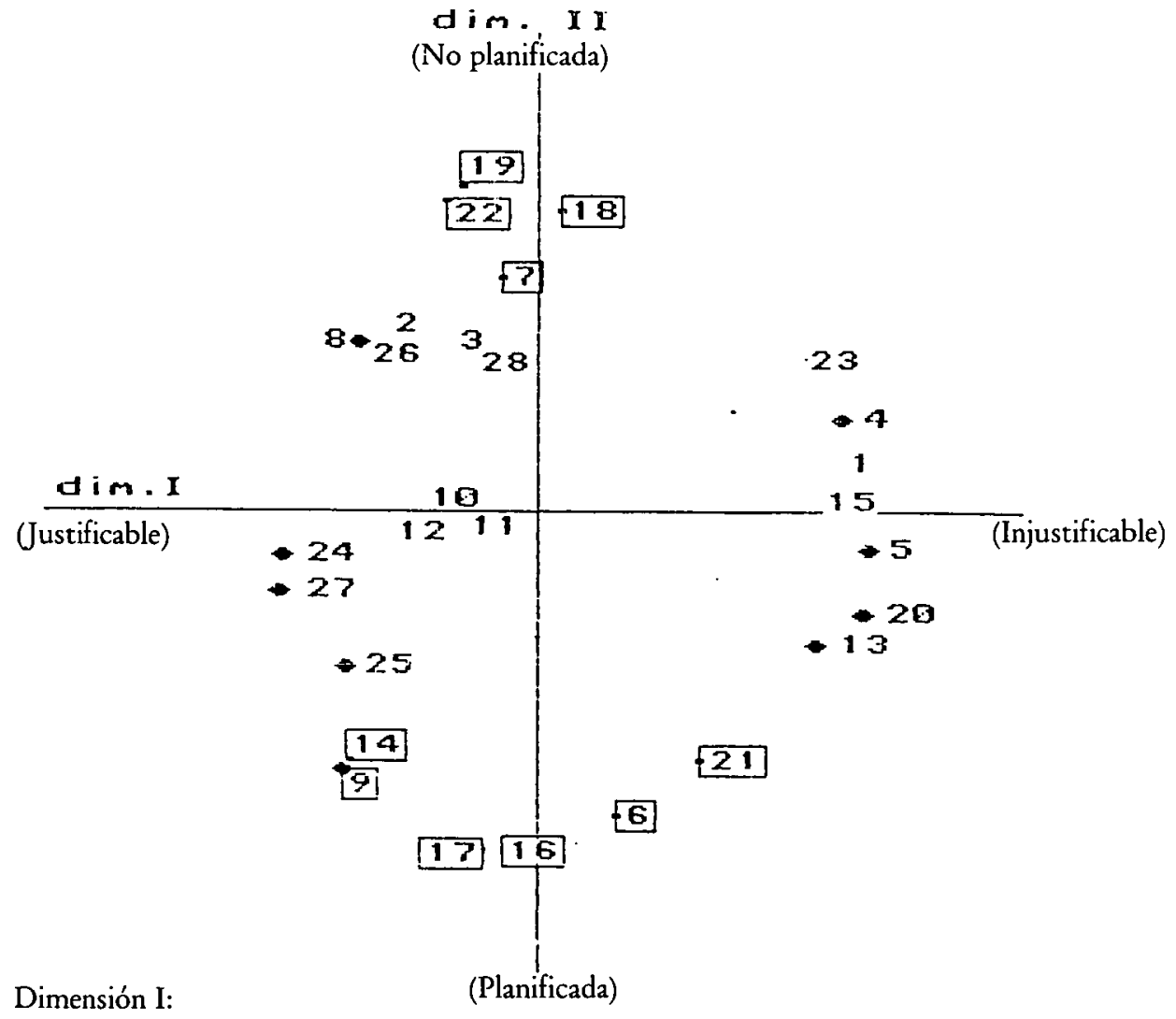

Dimensión II:

\begin{tabular}{clcl} 
Símbolo & Significado & Símbolo & \multicolumn{1}{c}{ Significado } \\
4 & Asalto a chicas & 17 & Alcohólico maltrata a su mujer \\
5 & Asalto anciano & 18 & Niños jugando matan un animal \\
6 & Violación novio & 19 & Discusión en el cine \\
7 & Insultos en bar & 20 & Asalto pareja de novios \\
8 & Golpes en el fútbol & 21 & Asalto chica \\
9 & Pelea en casa & 22 & Discusión en el autobús \\
13 & Intento secuestro & 25 & Padre en paro mata su ex-jefe \\
14 & Pela vecinos & 26 & Pela en el campo de fútbol \\
16 & Niños maltratados & 28 & Apuñalar a un compañero
\end{tabular}




\section{Figura 5}

Configuración de estimulos para las Dimensión III. Reclusos.

$$
\text { dim; III }
$$

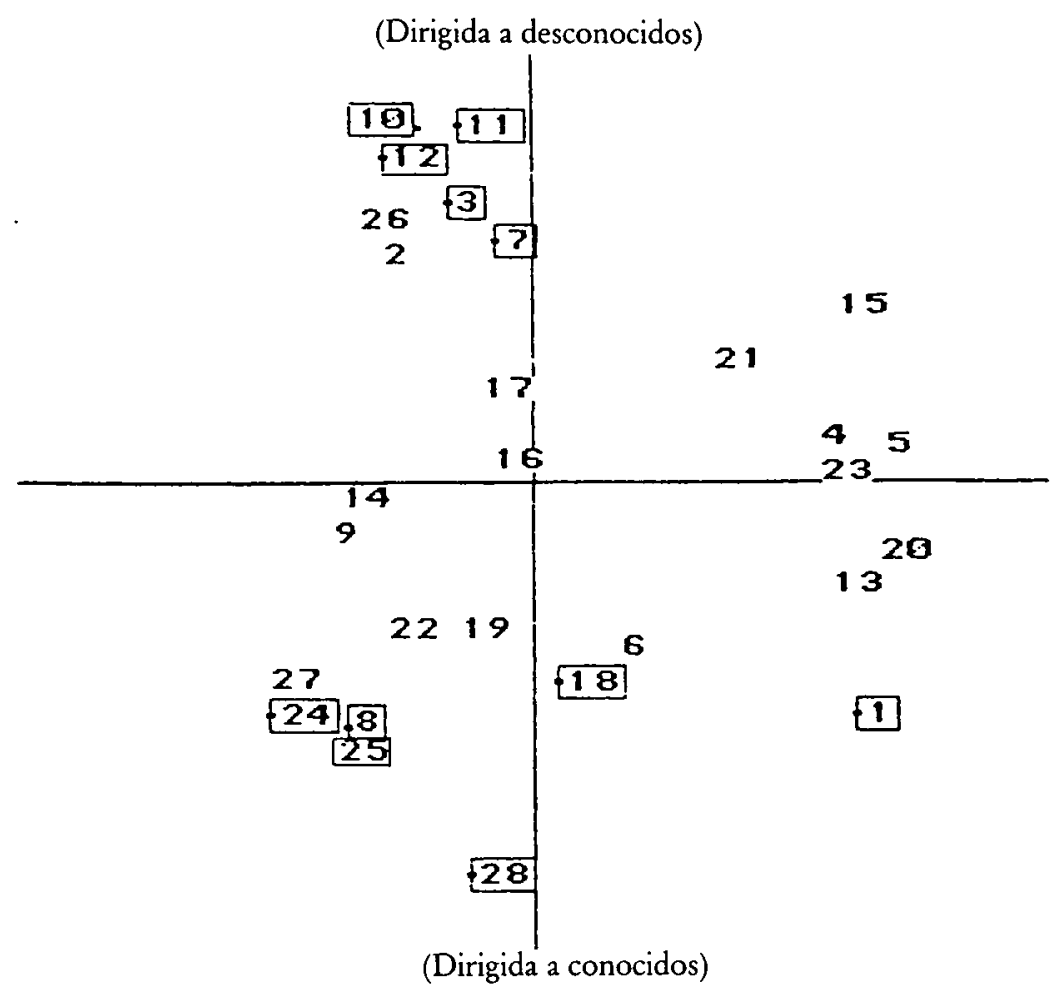

Dimensión III: Grado de implicación con la víctima

\begin{tabular}{clcl} 
Símbolo & \multicolumn{1}{c}{ Significado } & Símbolo & \multicolumn{1}{c}{ Significado } \\
1 & & 12 & Pelea en bar \\
3 & Pelea en discoteca & 18 & Niños matan un animal \\
7 & Por no pagar en un bar & 24 & Pelea entre hermanas en casa \\
8 & Pelea entre jugadores de fútbol & 25 & Padre en paro mata ex jefe \\
10 & Pelea en carretera & 28 & Ajuste de cuentas \\
11 & Pelea entre borrachos & &
\end{tabular}

Características de personalidad de los sujetos y su percepción de episodios agresivos

En un intento por conocer qué otro tipo de variables, además de las subculturales, pueden estar influyendo en la percepción de los episodios agresivos, los sujetos fueron evaluados en 9 variables de personalidad (señaladas anteriormente) que, en otros estudios conductuales, se mostraban relacionadas con la conducta agresiva. 
Para llevar a cabo este objetivo, se retomó el «espacio de sujetos» derivado por el programa ALSCAL para la muestra de universitarios, donde los pesos de cada sujeto, en cada una de las 4 dimensiones, constituyeron la variable de entrada para la serie de 9 Análisis Discriminantes.

El análisis de estos datos, realizado mediante el programa BMDP-7M de Dixon (1983), nos permitió concluir que los pesos de los sujetos, centrados en los grupos con puntuaciones altas o bajas en las 9 variables, no son significativamente distintos (con valores del estadístico $\mathrm{F}$ muy bajo y ninguno significativo $\mathrm{p}>$.05). De esto se desprende que las características de personalidad que hemos analizado no tienen ningún tipo de incidencia en la percepción que los sujetos universitarios tienen de los actos agresivos. Con la muestra de reclusos se realizó un procedimiento idéntico, alcanzándose los mismos resultados.

\section{DISCUSION FINAL}

Un aspecto importante que se deriva del presente estudio es que los episodios agresivos, que suceden naturalmente, se perciben en un dominio psicológico multidimensional. Sin duda, estos hallazgos suponen una evidencia favorable para aquellos autores que, de una u otra forma, abogan por un concepto «omnibus» de la agresión basado en la existencia de diferentes tipos de conductas agresivas.

Una vez puesto de manifiesto que los sujetos distinguen entre tipos de conductas agresivas, la segunda aportación de la investigación se sitúa en el intento por descubrir las dimensiones subyacentes que permitan diferenciar episodios agresivos. En relación a ello, los resultados obtenidos permitieron descubrir que los atributos de los episodios agresivos que configuran las dimensiones encontradas, son unos criterios prometedores para un sistema de clasificación. Esto fue puesto de manifiesto al comprobar que es posible generar una taxonomía empírica de los episodios agresivos combinando las dimensiones obtenidas. Y no sólo esto, sino que además, la nuestra, es una taxonomía que aglutina a las existentes. La taxonomía aquí propuesta constituye una visión completa e integradora del conjunto de taxonomías (teóricas y empíricas) propuestas hasta el momento por los distintos autores.

A continuación trataremos esto último más detalladamente. La clásica reducción de las características multifacéticas de los actos agresivos a la dimensión de gravedad (sobre todo en el campo de la Justicia) se refleja en la primera dimensión. En ella se matiza que una situación agresiva se evalúa como grave no por las consecuencias o daño de la acción, sino por la imposibilidad de la víctima a defenderse. En realidad, esta primera dimensión de gravedad, describe la tan manida «inseguridad ciudadana». Además, es una dimensión de carácter evaluativo y normativo en cuanto solamente es utilizada por la muestra de sujetos universitarios. Por tanto, la aportación a estos trabajos clásicos es que la valoración que hacen los sujetos de la agresión es más compleja de lo que se decía, necesita de 3 dimensiones más. Esto puede estar explicando, por ejemplo, los resultados de Sherman y Dowdle (1974) según los cuales la gravedad percibida no se relaciona con los castigos otorgados por la ley; o los resultados de Rosse y Press (1955) al señalar la existencia de una discrepancia entre las estimaciones populares y legales al juzgar un acto agresivo. 
La segunda dimensión, a diferencia de las otras, no refleja aspectos evaluativos o motivacionales, sino que distingue los aspectos externos de la acción. En concreto, si la acción se desarrolla entre personas conocidas o desconocidas, si la acción la comete el propio agresor, si estaba él solo o fue en presencia de otros, etc. Por ello fue definida como «implicación con la víctima». Esta dimensión recoge todas aquellas distinciones teóricas basadas en cuestiones externas tales como si fue física, verbal, directa, indirecta, individual o en grupo, etc.

La tercera dimensión aporta un dato interesante, el enorme conjunto de motivaciones destacadas por los autores parece que pueden reducirse a dos, instrumental y hostil. De ahí que la denominemos «instrumentalidad de la acción».

Asimismo, las otras dos dimensionones defendidas por Forgas et al. (1980) de probabilidad de ocurrencia, la de normativa encontrada por Campbell et al. (1985), la normativa-expresiva de Wolfgang y Ferracuti (1967), provocadano provocada de Zillman (1979), entre otras, parece que son características que se encuentran englobadas en nuestra $4 .{ }^{2}$ dimensión de «justibilidad». En concreto, esta dimensión agrupa a todos aquellos actos agresivos que están motivados por el deseo de adherirse a requisitos normativos que demandan agresión como una respuesta prescrita a la situación que está ocurriendo en ese momento.

Sin duda, estos datos, suponen un paso adelante en la controversia de cómo se puede denominar a cada conjunto de actos agresivos que comparten propiedades comunes. En la medida en que se incremente la homogeneidad de cada una de las clases de agresión, se habrá dado un paso importante para localizar los antecedentes sociales y psicológicos más importantes de tales actos. La presente investigación aconseja, que cualquier intento por ofrecer una adecuada taxonomía de cómo los sujetos perciben los diferentes episodios agresivos, ha detener en cuenta las 4 dimensiones recogidas en nuestro estudio: gravedad de la situación, grado de implicación con la víctima, instrumental-hostil y justificabilidad.

Con la presente investigación, también se pudo dar respuesta a las cuestiones planteadas en la introducción acerca de las influencias inter e intraculturales. Los sujetos universitarios utilizan 4 dimensiones para percibir un conjunto de episodios agresivos, mientras que los reclusos solamente utilizan 3 . De esto se deriva una primera interpretación ya que las diferencias vienen dadas por el uso o no de la dimensión de «gravedad de la situación». En nuestra opinión, esto pone de manifiesto un problema social de inseguridad ciudadana y, dado que los universitarios la padecen, necesitan de una dimensión que exceda el carácter evaluativo y que, a través de la cual, identifiquen una serie de conductas violentas, delictivas y repudiables. Creemos que esta dimensión está reflejando un atributo evaluativo de un fenómeno social temporal que padecen ciertos estamentos de la sociedad. Seguramente esta diferencia no se hubiese encontrado si en lugar de utilizar un grupo marginal (reclusos) se hubiese trabajado con otros grupos culturalmente diferentes. Ello hace que prefiramos interpretar las posibles diferencias en otros términos, por otra parte, ya señalados en el apartado de resultados.

Más concretamente, el análisis de los "espacios estimulares» aconsejan, por la razón señalada anteriormente, definir una sencilla estructura ortogonal en 3 dimensiones y plantear las diferencias subculturales más allá de la 
denominación de las dimensiones generales sino en el uso que los grupos hacen de ellas. De qué manera estos grupos sitúan a los episodios agresivos en lugares distintos. Los sujetos universitarios pueden entender que ciertas situaciones resulten provocadoras, pero en ningún momento justifican los asaltos, las violaciones, las venganzas, etc. Por el contrario, los delincuentes encarcelados (bajo la creencia de que es más importante valorar qué hizo la víctima con anterioridad, si se lo merece o no, etc.) incluso llegan a justificar que un padre en paro mate a su ex-jefe, o que un sujeto apuñale a un compañero por hacer trampas en el juego por entender que se adhieren a criterios normativos.

A nuestro juicio, estas diferencias encontradas ponen de manifiesto las diferencias reales entre 2 subculturas pertenecientes a una misma cultura, tal y como son los sujetos universitarios y reclusos españoles o, más concretamente, gallegos. Este último descubrimiento indica que si se desea comprender y predecir la conducta agresiva en determinadas muestras de sujetos, se debería tener en cuenta las dimensiones del conocimiento predominantes dentro de cada grupo o cultura.

En nuestra opinión, creemos necesario poner de manifiesto la importancia y necesidad de realizar trabajos similares, por ejemplo, en el ámbito jurídico. El hecho de que se haya comprobado que un mismo episodio agresivo (p. ej. «apuñalar a alguien») fuera evaluado por universitarios y reclusos de maneras tan distintas (grave y justificable, respectivamente), permite inferir que, en una muestra de profesionales de la Justicia, o en los Jurados, se producirían variaciones en la percepción de tales agresiones. Por ello reiteramos la necesidad de proseguir con este tipo de estudios empíricos en los que se tenga en cuenta las experiencias de socialización, estilos de vida, normas y valores de los sujetos para llegar a comprender cómo adquieren y cómo cambian en el tiempo tales percepciones de esas situaciones agresivas.

Por último, hacer una serie de consideraciones finales. En primer lugar, que el Escalamiento Multidimensional es una técnica de análisis adecuada para descubrir las dimensiones que los sujetos utilizan para percibir un conjunto de episodios agresivos. En segundo lugar, que nuestra taxonomía posee un valor teórico y empírico importante, por cuanto es una solución parsimoniosa $\mathrm{y}$, a la vez, aglutinadora de un buen conjunto de estudios taxonómicos (especialmente, el de Forgas et al., 1980 y el de Campbell et al., 1985) y, en tercer lugar, decir que la razón de no encontrar un consenso entre las taxonomías anteriores, quizá se deba a la variabilidad intercultural. Sin duda, para dar una contestación más firme a esta cuestión, sería de indudable valor replicar esta misma investigación, con igual procedimiento, en otras poblaciones tanto inter como intraculturalmente diferentes para conocer exactamente por qué resulta tan difícil de generalizar estos resultados. En concreto, serían de particular interés otras culturas (oriental, africana, gitana, etc.) así como los jueces, policía y otros sujetos que pudieran estar involucrados en la subcultura de la violencia. Creemos que estudios de este tipo permitirán poner de manifiesto la existencia de diferentes evaluaciones y percepciones de la conducta agresiva fruto de los distintos discursos y representaciones que sobre la misma existen en la sociedad en un momento determinado. 
Referencias:

ArCe, C. (1989). Escalamiento Multidimensional. En J. Arnau (1989): Métodos y Técnicas de Análisis de Datos en Ciencias del Comportamiento (en preparación).

ARCE, C. y Garling, Ti (1989). Multidimensional Scaling. Anuario de Psicologia, 43(4), 65-80.

ARCE, C. y ReAL, E. (1989). Una aplicación del Escalamiento Multidimensional al estudio de los mapas cognitivos en' ambientes a gran escala. Enviado para su publicación.

ArCe, C.; SeOAne, G. y Varela, J. (1989). Modelos Básicos en Escalamiento Multidimensional. Psicológica, 10, 35-48.

ATTILI, G. y HINDE, R. A. (1986). Categories of aggression and their motivational heterogenety. Etbology and Sociobiology, 7, 1, 17-27.

BandurA, A. (1973). Aggression: $A$ Social Learning Analysis. Englewood Cliffs, Nueva York: Prentice Hall.

Ber KOwITZ, L. (1962). Aggression. Nueva York: McGraw-Hill.

Berkowitz, L. (1969). Roots of Aggression. Nueva York: Atherton Press.

BERKowITZ, L. (1970). The contagion of violence: An S-R mediational analysis of some effects of observed aggresion. In W. I. Arnold y M. M. Page (Eds.): Nebraska. Symposium of Motivation. Lincioln: University of Nebraska Press.

Birnbacher, D. (1984). Three uses of the concept aggression. En A. Mummendey (Ed.): Social Psychology of Aggresion. Berlín: Springer-Verlag Berlin Heidelberg.

Buss, A. H. (1961). The Psychology of Aggression. Nueva York: Wiley.

CAmpBell, A.; MlunCER, S. y BiBel, D. (1985). Taxonomies of aggresive behavior: a preliminary report. Aggresive Behavior, 11, 217-222.

Carroll, J. D y Chang, J. J. (1970). Analysis of Individual Differences in Multidimensional Scaling Via an N-way generalization as «Eckhart-Young» decomposition. Psychometrika, 35, 283-319.

Christie, R, y GeIs, F. L. (1970). Studies in Macbiavelism. Nueva York: Academic Press.

Crowne, O. y Marlowe, D. (1960). A new scale of social desirability independent of psychopatology. Journal of Consulting Psychology, 24, 349-354.

Davison, M. L. (1983). Multidimensional Scaling. Nueva York: John Wiley Sons.

Dixon,, W. J. (1985). BMDP Statistical Software. Nueva York: University of California Press.

ENDLE, N, N. S. y HuNT, J. M. (1968). S-R inventories of hostility and comparisons of the proportions of variance from persons, responses and situations for hostility and anxiousness. Journal of Personality and Social Psychology, 9, 4, 309-315.

Ey SENCK, H. J. y EYSENCK, S. B. G. (1964). Manual of the Eysenck Personality Inventory. London: University of London Press.

FELSON, R. B. (1982). Impresion Management and the escalation of aggresion and violence. Social Psychology Quarterly, 45, 245-254.

Forgas, J. P.; Brown, L. L. y MenYhart, J. L. (1980). Dimensions of aggression: the perception of aggresive episodes. British Journal of Social and Clinical Psychology, 19, 215227.

GunN, J. (1973). Violence in Human Society. Nueva York: David Charles.

Henderson, M. (1986). An empirical classification of convicted violent offenders. British Journal Criminology, 22, 1-20.

JACKSON, D. N. y MESSIC, J. (1962). Responses styles and the assessment of psychopatology. En S. Messick y Ross (Eds.): Measurement in Personality and Cognition. Nueva York: Wiley.

Lorenz, K. (1966). On Aggression. Nueva York: Harcourt, Brace World.

OsGOOD, C. E. (1964). Semantic differential technique in the comparative study of cultures. American Antbropologist, 66 (3), 171-200.

Rose, A. M. y Press, A. E. (1955). Does the Punishment fit the Crime? a study in social evaluation. American Journal of Sociology, 61, 247-259.

SchoтT, F. (1971). What is Aggression? In H. Seling (Ed.): The Making of Human Aggression. Nueva York: St. Martin's Press.

Sherman, R. C. y Dowde, M. S. (1974). The percepcion of crime and punishment: A multidimensional scaling analysis. Social Science Research, 3, 109-126.

SHEPARD, S. R. (1972). A taxonomy of principal types of data and of multidimensional methods for their analysis. In R. N. Shepard, A. K. Romney y S. Nerlove (Eds.): Multidimensional Scaling: Theory and Applications in the Behavioral Sciences, 1, 21-47. Nueva York: Seminar Press.

SNYDER, M. 91974). The self-monitoring of expressive behavior. Journal of Personality and Social Psychology, 30, 526-537.

Takane, Y.; YounG, F. W. y Deleeuw, J. (1977). Non-metric Individual Differences Multidimensional Scaling: An Alternating least squares method with optimal scaling features. Psychometrika, 42, 7-67.

TEDESCHI, J. T. (1983). Social influence theory and aggression. In R. Green y E. Donnerstein (Eds.): Aggression: Theoretical and Empirical Reviewes. Nueva York: Academic Press.

Tосн, H. (1969). Violent Man: An inquiry into the Psychology of Violence. Chicago: Aldine. 
Varela, J. (1989). La Percepción de la Conducta Agresiva: Un Análisit' Multidimensional (Tesis doctoral), Universidad de Santiago de Compostela. España.

Varela, J. Arce, C. y Sabucedo, J. M. (1989). eEs Bidimensional la Percepción Social de la Agresión? Manuscrito remitido para su publicación.

Wish, M. y KaPLAN, S. (1977). Toward an implicit theory of interpiersonal Communication. Sociometry, 33, 4, 235-258.

Wish, M. DeUTSCH, M. y KafLan, S. (1976). Perceived dimensions of interpersonal relations. Journal of Personalty and Social Psychology, 33, 4.

WolfGAng, M. E. y FERRACuTI, F. (1967). The subculture of violence: Trowards and integrated theory in criminology. London: Tavistack.

Zillman, D. (1979). Hostility and Aggression. Nueva Jersey: Hillsdale Eribaum. 